\title{
From Quantum Cellular Automata to Quantum Field Theory
}

\author{
Alessandro Bisio* \\ Università degli studi di Pavia, Dipartimento di Fisica \\ E-mail: alessandro.bisio@unipv.it
}

This talk is based on Refs. [1,2,3]. We explore the idea that Quantum Field Theory could be grounded on a Quantum Cellular Automaton (QCA) model (i.e. a discrete unitary evolution of a lattice of quantum systems). In the 1+1-dimensional case, we show that the translation, parity and time-reversal symmetries lead to QCA model which recovers 1D Dirac field equation in the large scale limit. The discreteness of this model implies a breakdown of Lorentz covariance, which is replaced by a doubly special relativity model with an invariant lenght. Finally we will discuss the phenomenology of the QCA model and extensions to the three dimensional and interacting cases.

\section{References}

[1] A. Bisio, G. M. D'Ariano, A. Tosini Quantum Field as a Quantum Cellular Automaton: the Dirac free evolution in one dimension arXiv: 1212.2839

[2] A. Bisio, G. M. D'Ariano, A. Tosini Dirac quantum cellular automaton in one dimension: Zitterbewegung and scattering from potential Phys. Rev. A 88, 032301 (2013)

[3] A. Bibeau-Delisle, A. Bisio, G. M. D'Ariano, P. Perinotti, A. Tosini Doubly-Special Relativity from Quantum Cellular Automata arXiv:1310.6760

Frontiers of Fundamental Physics 14 - FFP14,

15-18 July 2014

Aix Marseille University (AMU) Saint-Charles Campus, Marseille

\footnotetext{
${ }^{*}$ Speaker.
} 